\title{
Endoscopic approach to the frontal sinus with modified lothrop technique. outcomes in a large Argentinean center
}

\begin{abstract}
Objectives: To determine the permeability of the frontal drainage obtained by the modified Lothrop surgical technique.

Study design: Descriptive and retrospective.

Methods: Patients treated with modified Lothrop technique to widening the frontal recess drainage pathway which was obstructed by inflammatory or tumor diseases in the Rhinology section of the Hospital Italiano in Buenos Aires were included between April 2011 and December 2017. Patients with minor permeabilizations were excluded (Draf I-II).

Results: 16 patients were treated, 7 women and 9 men, the youngest was 24 and the oldest 90 . The average age was 56 years. The etiologies of diseases affecting the frontal sinus were allergic fungal sinusitis $(2 / 16)$, recurred frontal mucocele with a history of Draf 2 (3/16), recurred frontal mucocele to external surgery with obliteration $(1 / 16)$, frontal mucocele, nasal polyposis and cystic fibrosis $(1 / 16)$, previous frontal sinusitis surgery (4/16), frontal mucopioceles $(2 / 16)$ and malignant tumors originating in the ethmoid and frontal sinus (3/16). An unilateral block drainage of the frontal by mucosal hyperplasia was diagnosed in a patient, caused by her allergic fungal rhinosinusitis 5 years after Lothrop surgery. Moreover the patient was operated again by transnasal approach permeabilizing the frontal sinus. Another patient had a total obstruction of the frontal drainage and was successfully repeated with the same surgical technique. The other patients had permeable frontal drainage and were asymptomatic during the average follow-up of 3.5 years.
\end{abstract}

Conclusion: The obtained permeability of the frontal sinus drainage with Lothrop modified technique was $87.50 \%(14 / 16)$. With rescue endonasal assisted surgery, frontal sinus patency was $100 \%$.

Keywords: frontal sinus, lothrop, draf III, frontal drainage, functional endoscopic sinus surgery, frontal recess, modified lothrop technique, paranasal sinuses, nose surgery, frontal sinus obstruction
Volume II Issue 2 - 2019

\author{
Carlos S Ruggeri,' Sebastian Aragon, ${ }^{2}$ Ana \\ Laura Cajelli, ${ }^{3}$ Lourdes Principe, ${ }^{2}$ Agustin \\ Martinez Font, ${ }^{2}$ Yesica Lijdens ${ }^{2}$ \\ 'Chief of Rhinosinusology division, Department of \\ Otolaryngology, Hospital Italiano de Buenos Aires, Argentina \\ ${ }^{2}$ Rhinosinusology division, Department of Otolaryngology, \\ Hospital Italiano de Buenos Aires, Argentina \\ ${ }^{3}$ Rhinosinusology fellowship, Department of Otolaryngology, \\ Hospital Italiano de Buenos Aires, Argentina
}

Correspondence: Carlos S Ruggeri, Chief of Rhinosinusology division, Department of Otolaryngology, Hospital Italiano de Buenos Aires, Argentina,

Email carlos.ruggeri@hospitalitaliano.org.ar

Received: October 08, 2018 | Published: April 02, 2019

\section{Introduction}

Obliteration of the frontal sinus, with previous dissection of an osteoplastic flap, was the treatment of choice in recurrent frontal sinusitis until 1990 . The reported failure rate is $10 \%$, but the incidence of complications is high (65\%). These may include: cerebrospinal fluid fistulas, supraorbital neuralgia, frontal osteitis, sepsis, depression or frontal prominence and mucocele. Lothrop described a combined procedure, performed externally and endonasally, with a resection of the floor of the frontal sinus and the intersinusal septum. It was successful in 29 of 30 patients, but the technique was considered very difficult by most of the surgeons of that time. Draf in 1991 and Gross in 1995, described a modification of the Lothrop technique, making the procedure entirely by endonasal route with endoscopes. Currently this surgical technique is the first choice to treat patients with frontal recurrent sinusitis, when other less complex endoscopic surgeries fail and also in severe diseases of the frontal sinus mucosa. The aim of this study is to determine the permeability of the frontal drainage obtained by the Modified Lothrop procedure.

\section{Materials and methods}

We performed a descriptive, retrospective study. All the patients treated with modified Lothrop Procedure to widening the frontal recess drainage pathway which was obstructed by inflammatory diseases or to expand the resection margin of a malignant rhinosinusal tumor, in the ENT department of the Italian Hospital of Buenos Aires between April 2011 and December 2017, were included.

We excluded patients treated with minor permeabilization of the frontal ostium (DRAF I-II).The surgeries were performed under general anesthesia. The surgical technique consisted of approaching the frontal sinus by endonasal route with $0^{\circ}$ and $30^{\circ}$ endoscopes. The frontal recess was dissected in both nasal cavities and by a DRAF I or IIA technique (dissection of the frontal recess/opening of the frontal recess to the middle turbinate) the frontal sinus ostium was identified. A bilateral anterior ethmoidectomy was performed.

In some patients, for the lateral technique, the anterior third of the middle turbinate was then resected bilaterally. The antero-superior sector of the nasal septum, between both ostiums, was removed creating a common cavity for drainage of the frontal sinus. The obtained limits were, laterally both lamina papyracea of the ethmoid bone, anteriorly the nasal bones and posteriorly the cribiform plate and posterior wall of the frontal sinus. Fig. 1 In other patients, the medial technique (outside-in) was performed: a mucosal flap was 
dissected anterior to the medial turbinate, on the frontal processes of the maxilla to identify the first olfactory nerve fiber. An anterior and superior septectomy, of approximately 1 centimeter, was made, and the frontal sinus was then approached in the most anterior part, by drilling the nasal bones. The drainage was extended to the posterior part of the sinus and laterally to both lamina papyracea. Medtronic Integrated Power Console lathe was used with diamond and cutting burrs of different diameters, curettes and a Kerrison-type rongeur. The mucosa of the lateral wall of the frontal sinus (lamina papyracea), responsible of the mucociliary transport, was preserved. A septal mucosal-free graft was used in some patients to cover the exposed bone anterior part of the sinus. The optical neuronavigation system was used in most of the patients (Figure 1, Figure 2).

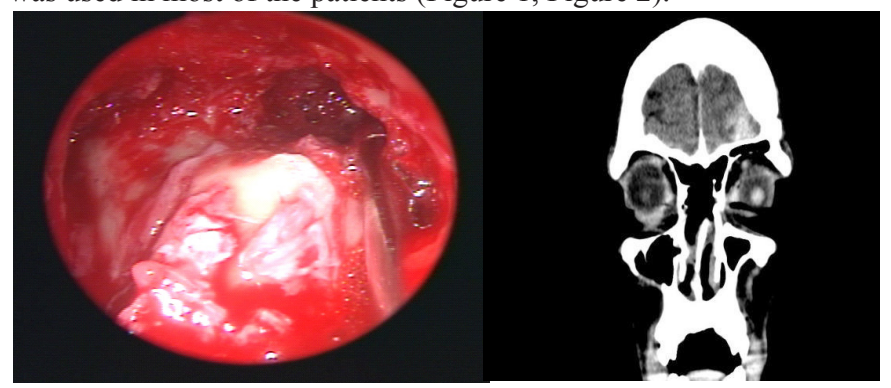

Figure I Modified Lothrop technique.

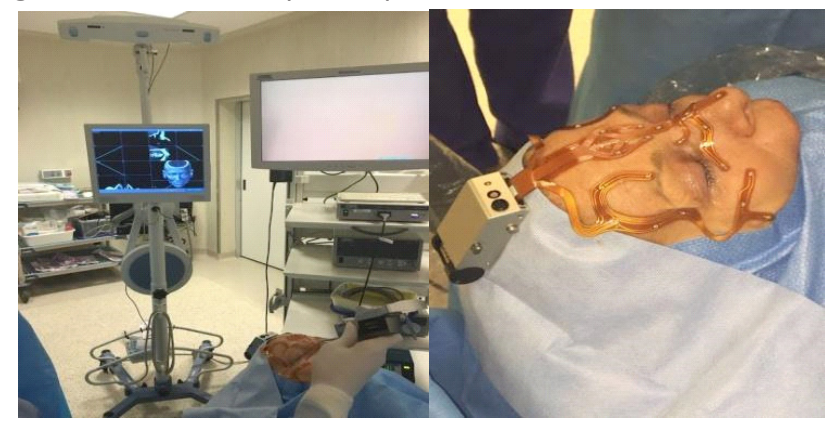

Figure 2 Optical neuronavigation systems.

The patients were followed weekly during the first month, with endonasal toillette. After 90days we evaluated the diameter of the frontal drainage obtained by videorhinofibroscopy. The surgery was considered effective when the patient had no more symptoms and the frontal drainage was endoscopically permeable. A computed tomography of the paranasal sinuses was requested to evaluate the permeability of the frontal drainage after the first year of surgery.

\section{Results}

Sixteen patients, seven women and nine men were treated, going from 24 to 90 years old (With an average age was 56 years). The etiologies of the diseases that affected the frontal sinus were: allergic fungal sinusitis (2/16), Recurrent frontal mucocele with a history of Draf 2 (3/16), frontal mucocele relapsed to external surgery with obliteration (1/16) frontal mucocele, nasal polyposis and cystic fibrosis (1/16), frontal sinusitis due to previous surgeries (4/16), frontal mucopioceles (2/16) and malignant tumors originating in the ethmoid and frontal sinus (3/16). Five patients had a history of transnasal surgeries with endoscopes: one was operated 11times and another three times for an allergic fungal rhinosinusitis. Another patient was operated on two opportunities because of a frontal mucoceles, and 2 patients underwent throw several endonasal surgeries and combined with external approach. Four had surgeries performed by external approaches, by mucoceles frontals, odontogenic myxoma and frontal sinusitis complication.

In three, the modified Lothrop technique was used to expand the margins of oncology resection due to malignant tumors that that compromised the recess and the frontal sinus. In one it was combined with an external approach. We used the optical neuronavigation system in three cases (Figure $3 \&$ Figure 4). In 12 patients, the sinus was approached through its ostiums (inside-out), and in 4 cases the frontal sinus was drained starting from the frontal recess floor in the midline (ouside-in). One patient had a cerebrospinal fluid fistula as a complication, which was detected and repaired with free grafts with multilayer technique in the same surgical time. One patient had unilateral frontal headaches 5 years after the Lothrop modified technique surgery. A unilateral blockage of frontal drainage was identified by mucosal hyperplasia caused by its allergic fungal sinuistis (Figure 5). It was operated again by transnasal route and the sinus was permeabilized. Another patient had a complete obliteration of the frontal drainage 1 year after surgery. It was operated again with modified Lothrop technique and it was possible to wide the frontal recess drainage pathway. The other patients had a permeable frontal drainage and were asymptomatic during the average follow-up time of 3.5years. The permeability of the frontal drainage obtained with modified Lothrop technique was $87.50 \%(14 / 16)$, and with rescue endonasal surgery, the permeability was $100 \%$.

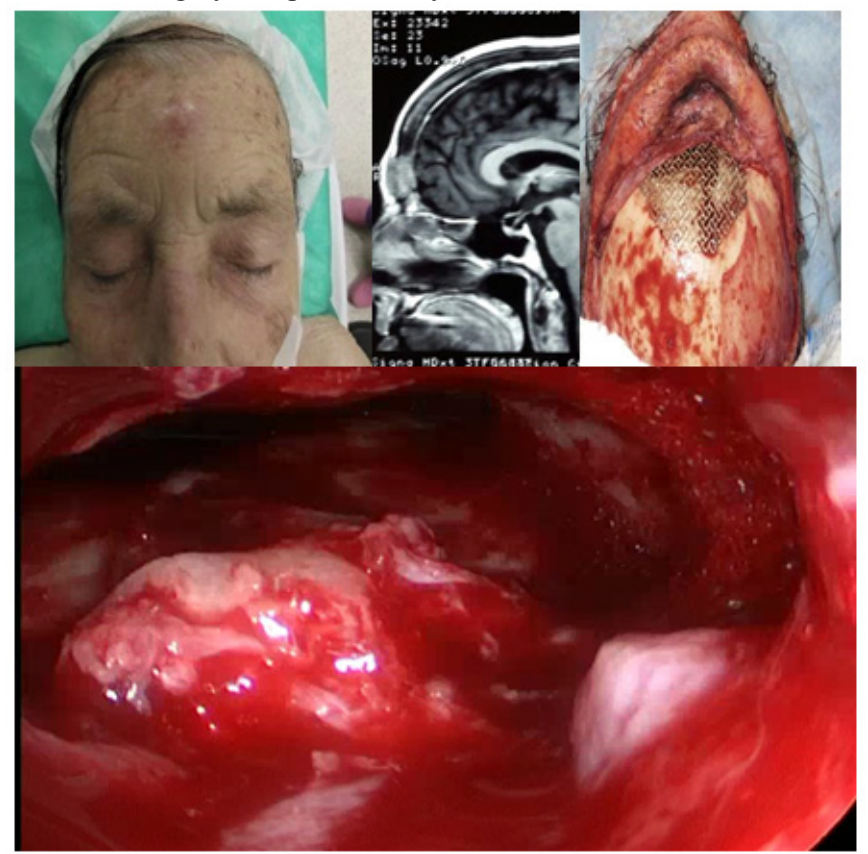

Figure 3 Epidermoid carcinoma of the frontal sinus. External approach+modified Lothrop procedure.

\section{Discussion}

The ideal surgical treatment of frontal sinus diseases would be the one that improves symptoms, cure the disease, preserve sinus function and produce minimal morbidity and deformity. In 1750 Runge performed a procedure of obliteration of the frontal sinus, and in 1870 Wells described an external and intracranial procedure to treat a mucocele. In the late 1800 s several surgeons treated frontal sinus diseases by radical procedures, resected the previous table, 
the mucosa and placed a stent. At the beginning of 1900, due to the cosmetic deformation and the high failure rate previous techniques, conservative ideas and attempts to solve pathologies emerged endonasal approaches. In 1914 Lothrop described a surgical technique to unclog the frontal drainage, combined an intranasal and external ethmoidectomy, and resected the floor of the frontal sinus and the superior sector of the nasal septum. The resection of the medial wall of the orbit caused the collapse of its contents and therefore blocking the frontal drainage. Moreover the lack of an adequate the lack of adequate visualization during the endonasal procedure made this technique not adopted by other surgeons of the time.

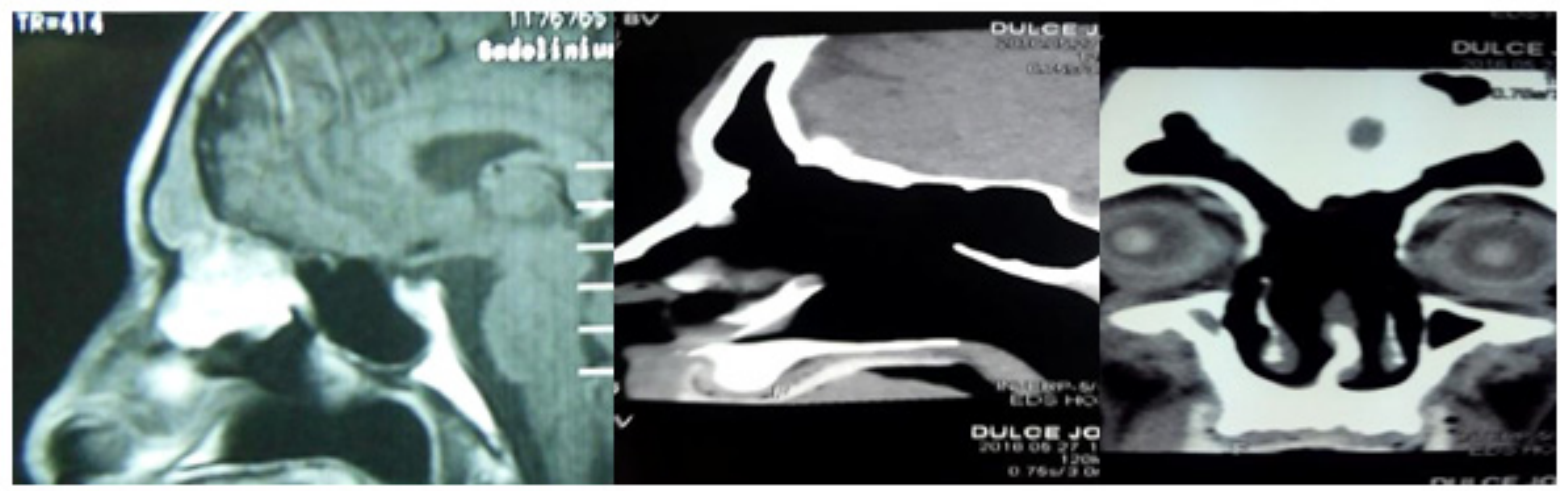

Figure 4 Undifferentiated carcinoma: Modified Lothrop procedure.

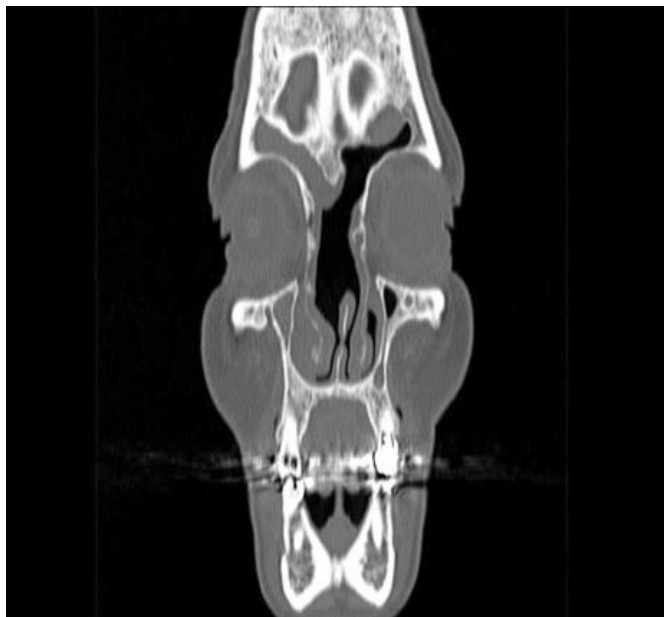

Figure 5 Frontal ostium obstructions due to mucosal Hiperplasy.

In 1949 Tato and Bergaglio described the osteoplastic surgery of the frontal sinus, and this was adopted as the gold standard surgery for many years. Hardy and Montgomery reported 10\% failures after performing this surgery. ${ }^{1}$ Weber diagnosed mucoceles by magnetic resonance imaging in $9.4 \%$ of patients treated with Osteoplastic technique with obliteration, after 2 years of follow-up., ${ }^{2,3}$ Draf systematized the frontal drainage according to the extension of the permeability obtained via endonasal: Draf I- anterior ethmoidectomy (the infundibulum and mucosa is not touched), Draf IIa-expansion of the frontal drainage between the papyracea lamina and the middle turbinate, Draf II-b: extension of the drainage between the papyracea lamina and the nasal septum, Draf III: expansion of the frontal drainage from one papyracea sheet to the other, drying the upper third of the nasal septum. In order to perform a modified Lothrop surgery, it must be previously evaluated in a computed tomography that there is an anteroposterior distance of at least $1.5 \mathrm{~cm}$ between the nasal bones and the anterior skull base. This technique is contraindicated in hypoplastic frontal sinuses. The approach can be lateral (inside-out), through the frontal recess (Draf 1-2) or medial, initiating the drilling behind the nasal bones in the midline (outside-in). ${ }^{4}$
This technique is recommended by Draf when there is already an anterior ethmoidectomy, there are no middle turbinates and there are few anatomical landmarks. The outside-in approach begins with the resection of the anterior-superior part of the septum nasal, and the dissection of mucosal flaps anterior to the middle turbinate, on the frontal apophyses of the maxilla, until identifying the first fiber of the olfactory nerve. Drill of the frontal sinus floor in the midline and enter the sinus behind the nasal bones, extending the drainage laterally, until the lamina papyracea and then to the posterior wall of the frontal sinus (approximately $7 \mathrm{~mm}$. ahead of the first fiber of the olfactory nerve). ${ }^{5}$ The floor of the frontal sinus can also be identified using the neuronavigation system and/or taking into account anatomical landmarks: posterior and superior to the most anterior and superior sector of the bony-cartilaginous junction of the septum, which is approximately at the level of the anterior sector of the middle turbinate and agger nasi. ${ }^{6}$ The indications of the modified Lothrop technique are all those cases in which it is not convenient to obliterate the frontal sinus: severe disease of the mucosa (Sampter's triad, recurrent nasal polyposis and fungal rhinosinusitis), very pneumatic frontal sinuses where the resection of the entire mucosa can be difficult, inverted papillomas and malignant tumors where obliteration could hide the diagnosis of recurrences, and failures after sinus obliteration. It is also convenient not to obliterate the sinus in frontal mucoceles that expands and the posterior wall posterior or inferior, where the mucosa is attached to the meninges or the orbit, making difficult to perform a complete resection of the mucosa and promotes the recurrence of mucoceles. Patients with chronic sinusitis with recurrent disease although previous surgeries with less complex endonasal techniques (Draf I-II) and as a previous step to endonasal or cranio-nasal craniectomies. One of the causes of failure of the Draf I-II techniques is bone neoformation due to osteitis of the frontal recess caused by surgery, which causes drainage stenosis. With the modified Lothrop technique, the frontal drainage is extended as much as possible, and if produces some degree of restenosis, this often does not affect the drainage of the sinus. In an animal study it was found that the ostium created by Lothrop surgery was reduced one third of its diameter, but this reduction did not affect the mucociliary drainage of the frontal. 
This happened during the first year after surgery, then the neoostium stabilized. ${ }^{7}$ The use of a graft free of septal mucosa, obtained when performing septectomy anterosuperior, and placed in the anterior margin of the frontal drainage would leave less bone exposed, and decrease the possibility of osteogenesis and obliteration of the Lothrop. Kountakis described healing rates with modified Lothrop in 2003, similar to those achieved with osteoplastic surgery, and Stankiewicz reported a $90 \%$ success in surgery rescue of osteoplastic failure, with modified Lothrop technique. ${ }^{8}$ Different studies describe the permeability rate of frontal drainage with modified Lothrop: Casiano reported that $57 \%$ of the patients controlled by rhinofibrolaryngoscopy with follow-up of 6.5 months had frontal permeability, and Gross reported $95 \%$ permeability to 12 months after surgery. ${ }^{9-11}$ Schlosser reported on 44 patients operated on with modified Lothrop, followed for 40 months that $9(20 \%)$ required a revision with a new Lothrop endoscopic procedure, and 8 of $44(18 \%)$ required osteoplastic surgery with obliteration. ${ }^{12}$ Wormald in 2003 published his results in 83 patients treated with modified Lothrop, obtained a primary permeabilization rate of $93 \%$ ( 77 of 83 patients) with a follow-up of 22months. ${ }^{13}$ In 2014, Wormald reported the results on 229 patients operated with the technique of Modified Lothrop with an average follow-up of 45 months. The surgery was satisfactory in the $95 \%$ ( 217 of 229 patients), the recurrence of the disease with persistence of symptoms and the need for surgical revision was given in 12 patients. Allergic fungal sinusitis and Staphylococcus aureus infections were risk factors for surgical failure. ${ }^{14}$ Recurrences may be due in many cases to the aggressiveness of the disease of the mucosa and not bone or scar obliteration, as in a patient of our series. Metson in a study of 204 patients operated on modified Lothrop, followed up average of 10.2 years described a reobstruction of the symptomatic frontal sinus of $29.9 \%(61 / 204)$. The surgeries performed by mucoceles and by tumors had a higher rate of failure (38.9\% and 58.3\%). Most of the surgery failures occurred during the first 2 years postoperatively, but some stenoses were observed up to 12 years after. ${ }^{15}$

Complications are infrequent, and are due to violation of the base of the skull, producing fistulas or pneumocephalus. Metson reported in a study of 204 patients treated with modified Lotthrop 4(1.9\%) intraoperative complications, due to bone opening of the base of the skull and dural tear, which produced cerebrospinal fluid fistulas. The defects were repaired successfully in the same surgical time with free grafts of mucous membrane. Eight patients (3.9\%) had epistaxis that required surgery. In our study there was a cerebrospinal fluid fistula, which was detected and repaired with a mucosal graft during the same surgery, and there were no epistaxis that required nasal tamponade or surgery. More common are minor complications such as easy-tocontrol epistaxis $(9 \%)$.

\section{Conclusion}

Conclusions: The obtained permeability of the frontal sinus drainage with Lothrop modified technique was $87.50 \%$ (14/16). With rescue endonasal assisted surgery, frontal sinus patency was $100 \%$.

\section{Acknowledgments}

None.

\section{Conflicts of interest}

Author declares that there are no conflicts of interest.

\section{References}

1. Hassan Ramadan. Hystory of Frontal Sinus Surgery. The Frontal Sinus. Stilianos Kountakis, Brent Senior, Wolfgang Draf, editors. SpringerVerlag, Berlin Heidelberg. 2005:1-6.

2. Weber R, Oraf W, KeerlR. Osteoplastic frontal sinus surgery with fat obliteration: technique and long results using magnetic resonance imaging in 82 operations. Laryngoscope. 2000;110(6):1037-1044.

3. Weber R, Draf W, Kratzsch B et al. Modern concepts of frontal sinus surgery. Laryngoscope. 2001;111(1):137-146.

4. David Chin, Kornkiat Snidvongs, Larry Kalish, et al. The Outsidein Approach to the Modified Endoscopic Lothrop Procedure. Laryngoscope. 2012;122(8):1661-1669.

5. Upadhyay S, Buohliqah L, Vieira G, et al. First Olfactory Fiber as an Anatomical Landmark for Frontal Sinus Surgery. Laryngoscope. 2016.126(5):1039-1045.

6. Lanza DC, Mc Laughlin RB, Hwang PH. The five year experience with endoscopic trans-septal frontal sinusotomy. Otolaryngol Clin North Am.2001;34(1):139-152.

7. Rajapaksa SP, Ananda A, Cain T, et al. The effect of the modified endoscopic Lothrop procedure on the mucociliary clearance of the frontal sinus in the animal model. Am Rhinol. 2004;18(4):183-187.

8. Kountakis Se, Gross CW. Long-term results of the Lothrop operation. Curr Opin Otolaryngol Head Neck Surg. 2003;11(1):37-40.

9. Casiano RR, Livingston JA. Endoscopic Lothrop procedure: The University of Miami experience. Am J Rhinol.1998;12(5):335-338.

10. Gross WE, Gross Cw, Becker D et al. Modifiedtransnasal endoscopic Lothrop procedure as an alternative to frontal sinus obliteration. Otolaryngol Head Neck Surg. 1995;113(4):427-434.

11. Gross CW, Schlosser RJ. The modified Lothrop procedure: Lessons learned. Laryngoscope. 2001;111(7):1302-1305.

12. Schlosser RJ, Zachmann G, Harrison $\mathrm{S}$ et al. The endoscopic modified Lothrop: Long-term follow up on 44 patients. Am J Rhinol. 2002;16(2):103-108.

13. Wormald PJ. Salvage frontal sinus surgery: The endoscopic modified Lothrop procedure. Laryngoscope. 2003;113(2):276-283.

14. Yuresh Naidoo BE, Ahmed Bassiouni, Mark Kenn. Long Term Outcomes for the Endoscopic Modified Lothrop/Draf III Procedure: A 10 Year Review. Laryngoscope. 2014;124(1):43-49.

15. Jonathan Y Ting, Arthur Wu, Ralph Metson. Frontal Sinus Drillout (Modified Lothrop Procedure): Long Term Results in 204 Patients. Laryngoscope. 2014;124(5):1067-1071. 